\title{
ANXIETY, DEPRESSION AND INFLUENCING FACTORS OF THE ELDERLY DISABLED UNDER THE INFLUENCE OF COVID-19 OUTBREAK: EVIDENCE FROM CHINA
}

\author{
Mu Li ${ }^{1,2}$, Yanping Yu ${ }^{1,2}$, Long Wang ${ }^{3} \&$ Zongyi Yin ${ }^{1,2}$ \\ ${ }^{1}$ School of Law, Humanities and Sociology, Wuhan University of Technology, Wuhan, China \\ ${ }^{2}$ China Disability Development Research Base, Wuhan University of Technology, Wuhan, China \\ ${ }^{3}$ School of Economics, Wuhan University of Technology, Wuhan, China
}

received: 25.2.2021;

revised: 8.7.2021;

accepted: 3.10 .2021

\section{SUMMARY}

Background: The elderly disabled have experienced serious negative emotions during the COVID-19 outbreak. However, the causes of anxiety and depression are not clear. This study aims to explore changes in mental states and influencing factors of the elderly disabled under the influence of the COVID-19 outbreak.

Subjects and methods: A total of 253 cases of elderly disabled in Wuhan, China were selected as the research group and observed from April to June 2020. Another 181 cases of elderly disabled in Yichang, China were observed from April to June 2020 and denoted Group A, while 100 cases of elderly disabled in Wuhan were investigated from August to November 2020 and denoted Group B. Another 100 cases of the elderly without disability were chosen as the control group. The Hamilton anxiety scale (HAMA) and Hamilton depression scale (HAMD) were used.

Results: HAMA and HAMD scores of the research group are higher than those of Groups $A$ and $B$ and the control group. HAMA and HAMD scores of Groups $A$ and $B$ are higher than those of the control group $(p<0.05)$. Solitude; pre-existing diseases; no stable and fixed friends; disability level-3 or -4; unmarried, divorced, or widowed; living in Wuhan; COVID-19 are risk factors for developing anxiety and depression in the elderly disabled after multivariate logistics regression $(p<0.05)$.

Conclusions: The incidence rate of anxiety and depression is very high in the elderly disabled under the influence of the COVID19 outbreak in Wuhan, China.

Key words: elderly disabled - anxiety - depression - COVID-19 outbreak

$$
* * * * *
$$

\section{INTRODUCTION}

Corona Virus Disease 2019 (COVID-19) is a largescale public health emergency that has threatened not only the life and health of people in Wuhan and worldwide but also caused considerable impacts on China's and the global economy while triggering extremely serious social and psychological problems (Jakovljevic et al. 2020, Lazzari et al. 2020, Chen et al. 2021). Existing studies have shown that psychological problems are common in disabled people ( $\mathrm{Li}$ et al. 2019, Miao et al. 2021). On the one hand, psychological problems will weaken the social ability of patients. On the other hand, disabilities will influence family harmony and reduce the living quality of patients (Morga et al. 2015). The disabled are a special and vulnerable group in China with limited capacity to participate in social activities due to poor physical conditions (Gao et al. 2018, Ren \& Guo 2020). This group of people is extremely susceptible to psychological disorders because of various challenges in employment and marriage. Travel behavior of the elderly disabled has been seriously influenced during the COVID-19 outbreak. Moreover, decreased social care for the elderly disabled can influence their confidence in life and thereby trigger serious negative emotions (Xin et al. 2016).
Anxiety and depression are two common negative emotions. Anxiety is a strong, excessive, and persistent feeling of worry and fear. Patients with anxiety usually demonstrate symptoms of malaise, cardiac acceleration, and dyskinesis. Depression is a major type of mood disorder and its clinical symptoms mainly include evident and persistent black mood and decreesing interests ( $\mathrm{Li}$ et al. 2020). The elderly disabled have been prohibited from attending normal outdoor activities and having normal contact with other people during the COVID-19 outbreak. Moreover, the inability of many elderly disabled living in solitude to care for themselves due to their weak actions has led to symptoms of anxiety and depression. Living environment, marriage condition, and physical condition are closely related to the development of anxiety and depression in the elderly disabled (Federici et al. 2017). However, the causes of negative emotions in this group of people during the COVID-19 outbreak remain unknown. Hence, investigating influencing factors of psychological health of the elderly disabled in Wuhan during the COVID-19 outbreak has become an important research topic in the field of psychology.

At present, China's government refers to people aged 60 or above as the elderly. Disabled people refer to people who cannot engage in certain activities in a normal way because of their unsound or missing func- 
tions, including psychology and human body structure (Liao 2020). Therefore, the elderly disabled is a special group of the elderly. Due to their physical or mental disabilities, the elderly disabled have brought many difficulties in their life. In addition, during the period of the COVID-19 outbreak, the elderly disabled are more likely to have many different mental health problems (Lee \& Kim 2020). It is very necessary to explore the mental health status of the elderly disabled, analyze the causes, and provide appropriate medical services and psychological intervention for the elderly disabled in the follow-up period, to integrate the elderly disabled s into the community and society during the period of COVID-19 outbreak.

Previous studies have explored the psychological problems of the elderly disabled. Kang et al. (2017) analyzed the formation mechanism of psychological problems of the elderly disabled, which provides a novel idea for the follow-up research. Due to the deterioration of brain function and the great changes in life before and after retirement, the elderly disabled have different degrees of mental health problems. The study of disabled elderly found that long-term bedridden and staying at home led to anxiety and depression, increased physical and mental health problems, and the level of mental health is significantly lower than that of the general elderly (Tajvar et al. 2018). With the aggravation of the disability degree, the self-evaluation of the elderly disabled has become negative, and the rate of social participation has gradually decreased. According to statistics, the average social participation rate of the elderly disabled in Japanese communities is only $26 \%$ (Kimura et al. 2013), and the elderly with severe disabilities can only passively participate in some community activities. In the last stage of life, most elderly disabled have difficulties in living independently and have been reduced to the edge of society, especially with serious psychological problems. Some studies believed that when the elderly disabled are facing psychological difficulties, the elderly should play a positive role in the family to create vitality and enjoy the life they have now, which can be used to solve the psychological difficulties faced by the elderly disabled (Hsu \& Tung 2010).

Relevant research data show that nearly $30 \%$ of the elderly suffer from obvious anxiety, depression, and other psychological disorders, $0.34 \%$ has certain symptoms of schizophrenia, and $0.75 \%$ has Alzheimer's disease ( Fu 2016). Affected by the COVID-19 outbreak, this kind of situation may be more serious in the elderly disabled. At the same time, the depression and suicide problems of the elderly group are increasingly prominent (Vrach \& Tomar 2020). The results of relevant research show that at present, more than half of the elderly show different degrees of depression and the rural areas are far higher than the cities, and the number of females is higher than that of men (Wu et al. 2010). At least 100000 elderly people commit suicide every year, which is the highest suicide rate in China (Wang 2013). Therefore, it is urgent to solve the mental health problems of the elderly disabled especially the attention of the elderly disabled in Wuhan, China during the period of the COVID-19 outbreak.

Overall, the psychological states of the elderly disabled in Wuhan during the COVID-19 outbreak were discussed to identify relevant influencing factors. This study aims to provide a reference for formulating intervention measures in the future.

\section{SUBJECTS AND METHODS}

\section{Participants}

Samples were collected from the Study Base of Disabled Career Development in Wuhan University of Technology. A total of 253 cases of the elderly disabled in Wuhan, China was investigated from April to June 2020 and chosen as the research group in this study. All cases conformed to the diagnosis of physical disability in the Manual of Disability Assessment (Federici et al. 2017). Meanwhile, another 181 cases of the elderly disabled in Yichang, China were observed from April to June 2020 and used as group A in this study, while 100 cases of the elderly disabled in Wuhan, China were investigated from August to November 2020 and denoted Group B. Another 100 cases of the aged ( $>60$ years old) without disability in the study area were selected as the control group. Patients with serious abnormal heart, liver, and kidney functions; hearing or vision disability; mental disorders; serious cognitive disorder; and severe malignant tumors were excluded from the study. All four groups show no significant differences in their general information $(p>0.05)$. The results are listed in Table 1.

Table 1. Comparison of general information of research objects

\begin{tabular}{|c|c|c|c|c|}
\hline General Information & Research Group & Group A & Group B & Control Group \\
\hline \multicolumn{5}{|l|}{ Gender } \\
\hline Male & 153 & 109 & 56 & 59 \\
\hline Female & 100 & 72 & 44 & 41 \\
\hline \multicolumn{5}{|l|}{ Age } \\
\hline$\geq 80$ & 112 & 86 & 41 & 42 \\
\hline$<80$ & 141 & 95 & 59 & 58 \\
\hline \multicolumn{5}{|l|}{ Living conditions } \\
\hline Solitude & 82 & 54 & 33 & 30 \\
\hline Living with family & 171 & 127 & 67 & 70 \\
\hline
\end{tabular}


Clinical data of all respondents, including age, gender, living conditions, pre-existing diseases, and time of disability, were collected via questionnaire. According to the Manual of Disability Assessment (Federici et al. 2017), disability in the aged group was divided into levels- $1,-2,-3$, and -4 . The test time of the survey was set $10-20 \mathrm{~min}$.

This study was approved by the ethical committee of the Research Base of Disabled Career Development in Wuhan University of Technology. All respondents were informed of the research content and objective of this study and signed written consent. Workers inputted data after the survey, and $10 \%$ of inputted questionnaires were checked with the original questionnaires to ensure the accuracy of inputted data.

\section{Measuring Tools}

\section{Hamilton Anxiety Scale (HAMA)}

This scale covers 14 terms of mental anxiety, tension, and somatic anxiety. Each term is scored from 0 to 4 , and the entire scale can obtain a score from 0 to 56 . A high score indicates severe anxiety, with the critical anxiety value at 28 . A patient suffers from anxiety if the score obtained is higher than 28 (Su \& Wang 2019).

\section{Hamilton Depression Scale (HAMD)}

This scale covers 14 terms, including depression, sense of guilt, and early insomnia. Each term is scored from 0 to 4 , and the entire scale can obtain a score from 0 to 56. A high score indicates severe depression, with the critical depression value at 28. A patient suffers from depression if the score obtained is higher 28 (Sunil et al. 2016).

\section{Statistical Analysis}

All research data were processed using SPSS 25. Enumeration data were expressed in percentage, and the intergroup difference was compared with the results of $\chi^{2}$ test. Measurement data were expressed in $(\overline{\mathrm{x}} \pm \mathrm{s})$ after normal test, and the intergroup difference was compared via $t$-test. The correlation between HAMA and HAMD scores of respondents was analyzed using the Pearson test. A multivariate logistics regression analysis on risk factors of developing anxiety and depression in the elderly was performed. $p<0.05$ indicates the statistical significance of the difference.

\section{RESULTS}

Comparison of four groups in HAMA and HAMD scores: HAMA and HAMD scores of the research group are higher than those of Groups A and B. HAMA and HAMD scores of Groups $\mathrm{A}$ and $\mathrm{B}$ are higher than those of the control group $(p<0.05)$. HAMA and HAMD scores of Groups A and B demonstrate no significant differences $(p>0.05)$. The results are listed in Table 2 .

Correlation analysis of HAMA and HAMD scores of patients: The HAMA score of the elderly disabled was positively correlated with the HAMD score $(r=0.392, p<0.001)$.

Comparison of general information between two groups: Incidence rates of depression and anxiety of the elderly disabled were $27.53 \%(147 / 534)$ and $33.90 \%$ (181/534), respectively. Incidence rates of risk factors of living in Wuhan; COVID-19 outbreak; solitude; preexisting diseases; no stable and fixed friends; disability level-3 or -4; and proportion of unmarried, divorced, or widowed were higher in the depression group compared with those of the no depression group $(P<0.05)$. Incidence rates of risk factors of living in Wuhan; COVID-19 outbreak; solitude; pre-existing diseases; no stable and fixed friends; disability level-3 or -4 ; and proportion of unmarried, divorced, or widowed were higher in the anxiety group compared with those of the non-anxiety group $(P<0.05)$. The results are presented in Table 3.

Risk factors that influence the anxiety of the elderly disabled: According to multivariate logistics regression, solitude; pre-existing diseases; no stable and fixed friends; disability level-3 or -4; unmarried, divorced or widowed; living in Wuhan; and COVID-19 are risk factors of developing anxiety in the elderly disabled $(p<0.05)$. The main reason may be that the elderly disabled living alone, without stable and fixed friends, divorced or widowed cannot solve the anxiety and fear of COVID-19. In addition, the elderly disabled generally have basic diseases, which will virtually compare their diseases with the symptoms of COVID-19, resulting in a heavier psychological burden and anxiety symptoms. On the other hand, there is disability levels-3 or -4 for the elderly, their actions and external expression may have some obstacles, their emotions cannot be effectively alleviated, psychological pressure is gradually increasing, which will produce more dangerous anxiety factors. The results are presented in Table 4.

Risk factors of developing depression in the elderly disabled: According to multivariate logistics regression analysis, solitude; no stable and fixed friends; disability level-3 or -4; unmarried, divorced, or widowed; living in Wuhan; and COVID-19 are risk factors of developing depression in the elderly disabled $(p<0.05)$. The emotional factors of depression are different from anxiety, even the escalation of anxiety. When anxiety can't be relieved in time, the elderly living alone, without stable and fixed friends, unmarried, divorced or widowed, who are in a state of anxiety for a long time, will change to the self-digestion of anxiety, and then keep silent, develop into depression tendency. Different from the formation of anxiety is whether the elderly have basic diseases. Basic diseases accelerate the elderly's worry about their self-state in a special period, which is not an important core point of causing selfdepression. Effective treatment of basic diseases alleviates the anxiety of the elderly disabled, while the attention to depression needs to pay more attention to the elderly living alone, marital status, and disability status. The results are listed in Table 5. 
Table 2. Comparison of HAMA and HAMD scores of four groups $(\overline{\mathrm{x}}+\mathrm{s}$, scores $)$

\begin{tabular}{lccc}
\hline Groups & $\mathrm{n}$ & HAMA & HAMD \\
\hline Research Group & 253 & $27.64 \pm 5.15$ & $25.69 \pm 5.02$ \\
Group A & 181 & $23.63 \pm 2.89^{* \#}$ & $22.82 \pm 2.73^{* \#}$ \\
Group B & 100 & $22.60 \pm 3.58^{* \#}$ & $22.05 \pm 3.17^{* \#}$ \\
Control Group & 100 & $11.08 \pm 2.33^{*}$ & $13.24 \pm 2.62^{*}$ \\
\hline${ }^{*} p<0.05$ in comparison with the research group; & ${ }^{*} P<0.05$ in comparison with the control group &
\end{tabular}

Table 3. Comparison of general information (cases) of two groups

\begin{tabular}{|c|c|c|c|c|c|c|c|c|c|}
\hline Indexes & $\begin{array}{l}\text { Number } \\
\text { of cases }\end{array}$ & $\begin{array}{l}\text { Dep-g* } \\
(n=147)\end{array}$ & $\begin{array}{l}\text { NDep-g* } \\
(\mathrm{n}=387)\end{array}$ & $\chi^{2}$ & $P$ & $\begin{array}{c}\text { Anx-g } \\
(n=181)\end{array}$ & $\begin{array}{l}\text { NAnx-g } \\
(n=353)\end{array}$ & $\chi^{2}$ & $P$ \\
\hline Age & & & & 0.872 & 0.350 & & & 0.518 & 0.472 \\
\hline$\geq 80$ & 239 & 61 & 178 & & & 83 & 190 & & \\
\hline$<80$ & 295 & 86 & 209 & & & 98 & 197 & & \\
\hline Gender & & & & 0.236 & 0.627 & & & 0.001 & 0.975 \\
\hline Male & 318 & 90 & 228 & & & 112 & 240 & & \\
\hline Female & 216 & 57 & 159 & & & 69 & 147 & & \\
\hline Living condition & & & & 169.381 & $<0.001$ & & & 194.975 & $<0.001$ \\
\hline Solitude & 169 & 109 & 60 & & & 139 & 64 & & \\
\hline Living with family & 365 & 38 & 327 & & & 42 & 323 & & \\
\hline Pre-existing diseases & & & & 38.791 & $<0.001$ & & & 16.403 & $<0.001$ \\
\hline Yes & 359 & 129 & 230 & & & 146 & 247 & & \\
\hline No & 175 & 18 & 157 & & & 35 & 140 & & \\
\hline Pension & & & & 0.073 & 0.787 & & & 0.607 & 0.436 \\
\hline Yes & 213 & 60 & 153 & & & 83 & 164 & & \\
\hline No & 321 & 87 & 234 & & & 98 & 223 & & \\
\hline Stable and fixed friends & & & & 5.54 & 0.019 & & & 7.755 & 0.005 \\
\hline Yes & 408 & 102 & 306 & & & 128 & 314 & & \\
\hline No & 126 & 45 & 81 & & & 53 & 73 & & \\
\hline Time of getting disabled & & & & 2.918 & 0.088 & & & 2.372 & 0.124 \\
\hline Congential & 139 & 46 & 93 & & & 63 & 110 & & \\
\hline Acquired & 395 & 101 & 294 & & & 118 & 277 & & \\
\hline Preretirement work & & & & 0.121 & 0.728 & & & 1.313 & 0.252 \\
\hline Intellectual work & 136 & 39 & 97 & & & 60 & 110 & & \\
\hline Physical work & 398 & 108 & 290 & & & 121 & 277 & & \\
\hline Education background & & & & 0.218 & 0.64 & & & 0.005 & 0.943 \\
\hline Junior high school or lower & 212 & 56 & 156 & & & 78 & 168 & & \\
\hline Senior high school or higher & 322 & 91 & 231 & & & 103 & 219 & & \\
\hline Disability level & & & & 125.492 & $<0.001$ & & & 201.11 & $<0.001$ \\
\hline Levels- 1 and -2 & 395 & 58 & 337 & & & 69 & 360 & & \\
\hline Levels-3 and -4 & 139 & 89 & 50 & & & 112 & 27 & & \\
\hline Marriage & & & & 121.404 & $<0.001$ & & & 135.101 & $<0.001$ \\
\hline Married & 436 & 76 & 360 & & & 101 & 369 & & \\
\hline Unm-di-wid* & 98 & 71 & 27 & & & 80 & 18 & & \\
\hline Living area & & & & 5.859 & 0.015 & & & 18.213 & $<0.001$ \\
\hline Yichang & 181 & 38 & 143 & & & 35 & 146 & & \\
\hline Wuhan & 353 & 109 & 244 & & & 146 & 247 & & \\
\hline Survey period & & & & 4.486 & 0.034 & & & 15.902 & $<0.001$ \\
\hline COVID-19 outbreak & 434 & 128 & 306 & & & 166 & 302 & & \\
\hline Non-COVID-19 outbreak & 100 & 19 & 81 & & & 15 & 85 & & \\
\hline
\end{tabular}

*Legend: Unm-di-wid: Unmarried, divorced, or widowed; Dep-g: Depression group; NDep-g: Nondepression group;

Anx-g: Anxiety group; NAnx-g: Nonanxiety group

Table 4. Risk factors of anxiety

\begin{tabular}{lccc}
\hline Factors & OR value & $95 \%$ CI & $P$-value \\
\hline Living conditions (solitude vs. living with family members) & 1.765 & $1.081 \sim 2.881$ & 0.024 \\
Pre-existing diseases (Yes vs. No) & 1.649 & $1.010 \sim 2.691$ & 0.046 \\
Stable and fixed friends (Yes vs. No) & 1.818 & $1.114 \sim 2.968$ & 0.017 \\
Disability level (levels-1 and -2 vs. levels-3 and -4) & 1.831 & $1.122 \sim 2.989$ & 0.016 \\
Marriage (married vs. unmarried, divorced, and widowed) & 1.998 & $1.224 \sim 3.261$ & 0.006 \\
Survey period (COVID-19 outbreak vs. non-COVID-19 outbreak) & 1.859 & $1.139 \sim 3.034$ & 0.014 \\
Living area (Yichang vs. Wuhan) & 2.034 & $1.246 \sim 3.320$ & 0.005 \\
\hline
\end{tabular}


Table 5. Risk factors of depression

\begin{tabular}{|c|c|c|c|}
\hline Factors & $O R$ value & $95 \% C I$ & $P$-value \\
\hline Living conditions (solitude vs. living with family members) & 1.665 & $1.020 \sim 2.718$ & 0.042 \\
\hline Pre-existing diseases (Yes vs. No) & 1.522 & $0.932 \sim 2.484$ & 0.094 \\
\hline Stable and fixed friends (Yes vs. No) & 1.714 & $1.050 \sim 2.798$ & 0.032 \\
\hline Disability level (levels- 1 and -2 vs. levels-3 and -4 ) & 1.804 & $1.105 \sim 2.945$ & 0.019 \\
\hline Marriage (married vs. unmarried, divorced, and widowed) & 1.859 & $1.139 \sim 3.034$ & 0.014 \\
\hline Survey period (COVID-19 outbreak vs. non-COVID-19 outbreak) & 1.893 & $1.160 \sim 3.089$ & 0.011 \\
\hline Living area (Yichang vs. Wuhan) & 1.835 & $1.124 \sim 2.995$ & 0.016 \\
\hline
\end{tabular}

\section{DISCUSSION}

Table 1 shows that incidence rates of depression and anxiety in the elderly disabled are $27.53 \%$ and $33.90 \%$, respectively. HAMA and HAMD scores of the research group were higher than those of Groups A and $\mathrm{B}$ and the control group. HAMA and HAMD scores of Groups A and B are higher than those of the control group. These findings reflected that the elderly disabled in Wuhan are susceptible to anxiety and depression during the COVID-19 outbreak. Such a risk is higher than that of respondents from Yichang and Wuhan during the non-COVID-19 outbreak mainly because residents of Wuhan were isolated during the COVID-19 outbreak. Both travel restrictions and city blocking during this period influenced the daily life of the elderly disabled. Moreover, the incubation period of COVID-19 is long and the number of diagnosed COVID-19 cases in Wuhan increases daily. Patients between normal and risk states are highly susceptible to anxiety attacks because they fail to accept their existing health conditions (Yukhnenko et al. 2020). COVID-19 outbreak causes significant impacts on public psychology (Jing \& Wang 2020, Pillay \& Barnes 2020).

As shown in Table 2, HAMA and HAMD scores are positively related to the degree of anxiety and depression of the elderly disabled mainly because anxiety can cause energy loss and excessive worrying of patients that influence their sleep quality and daily lives and likely result in the development of depression symptoms. Mao et al. (2018) analyzed the elderly disabled and found that living in solitude increases the risk of negative emotions in the elderly. The elderly disabled project their life pressures to family members due to the decreased self-care ability (Lamb 2019). However, the necessity of respondents living in solitude to take care of themselves daily increases their life burden and susceptibility in developing anxiety and fears (Huang et al. 2019, Dennis et al. 2021).

Table 3 shows differences between depression and no depression groups as well as anxiety and nonanxiety groups in terms of living in Wuhan; COVID19 outbreak; solitude; pre-existing diseases, no stable and fixed friends; disability level-3 or -4 , and proportion of unmarried, divorced, or widowed. These findings revealed that the development of anxiety and depression in the elderly disabled may be related to living in Wuhan and COVID-19 (19). Xue et al. (2017) pointed out that living in solitude and low family income are causes of negative emotions in the elderly disabled. Family income level is an important index that reflects the economic capability of a person (Batty et al., 2020). Dependence on other family members increases gradually with the increasing age of the elderly disabled. The complete reliance of the elderly disabled without a pension and labor ability on their children for support will increase family expenditures. The instability of income along with difficulties in performing outdoor activities and rehabilitation during the COVID-19 outbreak has caused serious economic pressure in Wuhan. Meanwhile, the absence of income for the elderly disabled exerts strong psychological pressure on them. The quality of life for many of the elderly has declined because of their inability to acquire the latest information due to their lack of skill in using technology, and this sense of uncertainty caused by the COVID-19 pandemic has seriously influenced the psychological state of the elderly disabled (Wang et al. 2020).

The multivariate logistics regression analysis is shown in Tables 4 and 5 further proves that solitude; no stable and fixed friends; disability level-3 or -4 ; unmarried, divorced, or widowed; living in Wuhan; COVID-19 are causes of anxiety and depression in patients. Therefore, the intervention of these factors can reduce the risk of anxiety and depression.

Some limitations of this study should be mentioned. Undoubtedly, the difficulties in communication to the elderly disabled can bring obstacles to having representative samples. Also, self-reporting without the possibility of an objective assessment by professionals. As a result, the quantity and quality of research samples are limited. In addition, with the continuous spread of the COVID-19 epidemic, how to alleviate the anxiety and depression of the elderly disabled can be further explored in the follow-up study.

\section{CONCLUSION}

To explore changes in mental states and influencing factors of the elderly disabled under the influence of the COVID-19 outbreak, the Hamilton anxiety scale (HAMA) and Hamilton depression scale (HAMD) were used to evaluate the elderly disabled in Wuhan, 
China, and to analyze the risk factors of depression and anxiety. The following conclusions are obtained: the high incidence rate of anxiety and depression in the elderly disabled is caused by solitude; no stable and fixed friends; disability level-3 or -4; unmarried, divorced, or widowed; living in Wuhan; and COVID19. Hence, developing social services and decreasing the economic pressure on other family members is suggested to help the elderly disabled and family members suffering from anxiety and depression.

\section{Acknowledgements:}

This study was supported by the Fundamental Research Funds for the Central Universities of China (No. WUT2020VI058).

\section{Conflict of interest: None to declare.}

\section{Contribution of individual authors:}

Mu Li: Conception and design, analysis.

Yanping Yu: Planning and designing data collection, statistical analysis.

Long Wang: Material preparation, data collection.

Zongyi Yin: Draft preparation, approval of the final version.

All authors have read and agreed to the published version of the manuscript.

\section{References}

1. Batty GD, Deary IJ, Luciano M: Psychosocial factors and hospitalisations for COVID-19: Prospective cohort study based on a community sample. Brain Behavior Iтmu 2020; 17:31309-31312

2. Chen G, Gong J, Qi Z, Zhong S, Su T, Wang J, Fu S, Huang L, Wang Y: The psychological status of general population in Hubei province during the COVID-19 outbreak: A cross-sectional survey study. Frontiers in Public Health 2021; 9:622762

3. Dennis D, Radnitz C, Wheaton MG: A perfect storm? Health anxiety, contamination fears, and COVID-19: Lessons learned from past pandemics and current challenges. International Journal of Cognitive Therapy 2021. https://doi.org/10.1007/s41811-021-00109-7

4. Federici S, Bracalenti M, Meloni F, Luciano JV: World Health Organization disability assessment schedule 2.0: An international systematic review. Disability and Rehabilitation 2017; 39:2347-2380

5. Fu SL: Mental health self-assessment and influence factors analysis among different age elderly. Journal of Guangdong University of Technology (Social Sciences Edition) 2016; 16:20-26

6. Gao JM, He P, Zheng XY: Analysis on the influencing factors of self-care ability of hearing disabled elderly in China. Chinese Journal of Public Health 2018; 34:337-341

7. Hsu HC, Tung HJ: What makes you good and happy? Effects of internal and external resources to adaptation and psychological well-being for the disabled elderly in Taiwan. Aging \& Mental Health 2010; 14:851-860
8. Huang Y, Huang Y, Bao M: Psychological resilience of women after breast cancer surgery: a cross-sectional study of associated influencing factors. Psychology Health \& Medicine 2019; 24:866-878

9. Jakovljevic $M$, Bjedov $S$, Jaksic $N$, Jakovljevic I: COVID-19 Pandemia and Public and Global Mental Health from the Perspective of Global Health Security. Psychiatr Danub 2020;32:6-14

10. Jing G, Wang CM: The impact of the "new coronavirus COVID-19" epidemic on the public's psychology and its protection. Psychology Monthly 2020; 15:1-2

11. Kang HJ, Bae KY, Kim SW, Shin HY, Shin IS, Yoon JS, Kim JM: Impact of anxiety and depression on physical health condition and disability in an elderly Korean population. Psychiatry Investigation 2017; 14:240-248

12. Kimura M, Yamazaki S, Haga H, Yasumura S: The prevalence of social engagement in the disabled elderly and related factors. ISRN Geriatrics 2013; 2013:709823

13. Lamb S: On being (not) old: Agency, self-care, and lifecourse aspirations in the United States. Medical Anthropology Quarterly 2019; 33:263-281

14. Lazzari C, Shoka A, Nusair A, Rabottini M: Psychiatry in Time of COVID-19 Pandemic. Psychiatr Danub 2020; 32:229-235

15. Lee S, Kim J: A country report: impact of COVID-19 and inequity of health on South Korea's disabled community during a pandemic. Disability \& Society, 2020; 35:1514-1519

16. Li J, Yang Z, Qiu H, Wang Y, Jian L, Ji J, Li K: Anxiety and depression among general population in China at the peak of the COVID-19 epidemic. World Psychiatry 2020; 19:249-250

17. Li YZ, Zhang YP, Xiong M: Characteristics and influencing factors of psychological resilience of the elderly disabled. Chinese Journal of Behavioral Medicine and Brain Science 2019; 28:354-358

18. Liao J: The quota system for employment of people with disabilities in China: Policy, practice, barriers, and ways forward. Disability \& Society 2020; 36:326-331

19. Mao P, Feng H, Xu S: Well-child care delivery in the community in China: Related factors and quality analysis of services. Plos One 2018; 13:e0190396

20. Miao C, Humphrey RH, Qian S: Emotional intelligence and servant leadership: $A$ meta-analytic review. Business Ethics: A European Review 2021; 30:231-243

21. Morga P, Traczyk J, Wittenbeck K: Review of research on the impact of physical activity on mental state of the elderly. Physiotherapy 2015; 23:42-53

22. Pillay AL, Barnes BR: Psychology and COVID-19: impacts, themes and way forward. South African Journal of Psychology 2020; 50:148-153

23. Ren FF, Guo RJ: Public Mental Health in Post-COVID19 Era. Psychiatr Danub 2020; 32:251-255

24. Su SW, Wang D: Health-related quality of life and related factors among elderly persons under different aged care models in Guangzhou, China: a crosssectional study. Quality of Life Research 2019; 28:12931303

25. Sunil KR, Vishav C, Sujeet R: Feasibility of using everyday abilities scale of India as alternative to mental state examination as a screen in two-phase survey estimating the prevalence of dementia in largely illiterate Indian population. Indian Journal of Psychiatry 2016; 58:459-461 
26. Tajvar M, Grundy E, Fletcher A: Social support and mental health status of older people: a population-based study in Iran-Tehran. Aging \& Mental Health 2018; 22:344-353

27. Vrach IT, Tomar R: Mental health impacts of social isolation in older people during COVID pandemic. Progress in Neurology and Psychiatry 2020; 24:25-29

28. Wang WL: Suicide of the elderly population in China. Population and Development 2013; 19:83-89

29. Wang Y, Duan Z, Ma Z: Epidemiology of mental health problems among patients with cancer during COVID-19 pandemic. Translational Psychiatry 2020; 10:263-265

30. Wu XL, Li J, Wang LL: Analysis on the depressive symptoms of elderly in China. Population Journal 2010; $5: 43-47$

31. Xin YZ, Yin NM, Ya FT: 965 Analysis of vision and related risk factors among the falls of elderly in communities of Beijing city, China. Injury Prevention 2016; 22:343-344

32. Xue $F Y$, Zhe $Y U$, Cheng $Z B$ : A study on subjective wellbeing status and influencing factors among old people in Hangzhou City. Preventive Medicine 2017; 29:433-437

33. Yukhnenko D, Blackwood N, Fazel S: Risk factors for recidivism in individuals receiving community sentences: a systematic review and meta-analysis. CNS Spectrums $2020 ; 25: 252-263$

Correspondence:

Zongyi Yin, Lecturer, PhD

School of Law, Humanities and Sociology, Wuhan University of Technology

China Disability Development Research Base, Wuhan University of Technology

\#205 Luoshi Road, Hongshan District, 430070 Wuhan, China

E-mail: yinzongyi1984@163.com 